\title{
Developing the concept of dietary estimation of fermentable carbohydrate (FC)
}

\author{
S. Alharbi ${ }^{1}$, A. L. Garcia ${ }^{1}$, D. J. Morrison ${ }^{2}$, L. Hanske ${ }^{1}$ and C. A. Edwards ${ }^{1}$ \\ ${ }^{1}$ Human Nutrition, School of Medicine, College of Medical, Veterinary \& Life Sciences University of Glasgow, \\ New Lister Building, Glasgow Royal Infirmary, 10-16 Alexandra Parade, Glasgow, G31 2ER, Scotland, \\ United Kingdom and ${ }^{2}$ SUERC, University of Glasgow, East Kilbride, United Kingdom, G75 0QF
}

Dietary fibre is believed to have many health benefits (1) but confirming its associations with reduction in risk of chronic diseases is difficult as different fibres have a variety of possible actions including the production of short chain fatty acids (SCFA) during colonic fermentation. There are also several fermentable substrates that have not been included in dietary fibre estimates for epidemiological studies. Thus developing methods to estimate fermentable carbohydrates in the diet would aid better characterisation of the impact of fermentation on health. Using evidence in the literature we developed several possible equations to estimate the amount of potential fermented carbohydrate in the diet and tested these against SCFA measured in the urine of 122 healthy subjects on their normal diet.

Equations to estimate fermentable carbohydrate (FC) were based on content of dietary fibre (DF) and non-starch polysaccharide (NSP), a proportion of total starch (TS) and non- digestible oligosaccharide content. These were then applied to 3 day weighed dietary records using data from food tables and other published sources. These values were correlated with the SCFA concentrations in 24 hour urine collections measured by GC-MS.

The relationships were examined for 78 subjects after excluding those who were shown to be under-reporting dietary intake using a physical activity level cut-off of 1.2. The Spearman correlation coefficient (r) and significance (p) values for the correlations with urinary acetate are shown in Table 1 for dietary fibre (AOAC 991.43 method) and NSP and for two corresponding equations, one including no insoluble fibre and the other $50 \%$ insoluble fibre (Figure 1). There was no significant relationship with the other SCFA.

Table 1. Associations between fermentable carbohydrate estimations using different equations and urinary SCFA

\begin{tabular}{|c|c|c|c|c|c|c|c|c|c|c|c|c|}
\hline \multirow[b]{2}{*}{$n=78$} & \multicolumn{2}{|l|}{$\mathrm{DF}$} & \multicolumn{2}{|c|}{ FC-DF $1^{¥}$} & \multicolumn{2}{|c|}{ FC-DF $2^{\S}$} & \multicolumn{2}{|l|}{ NSP } & \multicolumn{2}{|c|}{ FC-NSP $1^{\text {}}$} & \multicolumn{2}{|c|}{ FC-NSP $2^{Л}$} \\
\hline & $\bar{r}$ & $\mathrm{p}$ & $\mathrm{r}$ & $\mathrm{P}$ & $\mathrm{r}$ & $\mathrm{p}$ & $\mathrm{r}$ & $\mathrm{p}$ & $\mathrm{r}$ & $\mathrm{p}$ & $\mathrm{r}$ & $\mathrm{p}$ \\
\hline Acetate $24 \mathrm{~h}$ & $0 \cdot 13$ & NS & $0 \cdot 20$ & NS & $0 \cdot 22$ & 0.04 & $0 \cdot 14$ & NS & $0 \cdot 27$ & 0.01 & $0 \cdot 26$ & 0.02 \\
\hline
\end{tabular}

$¥ \mathrm{FC}-\mathrm{DF} 1=$ soluble DF + oligosaccharide $+10 \% \mathrm{TS} ;{ }^{\text {}} \mathrm{FC}-\mathrm{NSP} 1=$ soluble NSP + oligosaccharide $+10 \% \mathrm{TS}$

$\S$ FC-DF2 $=$ soluble DF $+50 \%$ insoluble DF + oligosaccharide $+10 \%$ TS; ЛFC-NSP2 $=$ soluble NSP $+50 \%$ insoluble NSP + oligosaccharide $+10 \%$ TS; $($ Spearman's correlation)
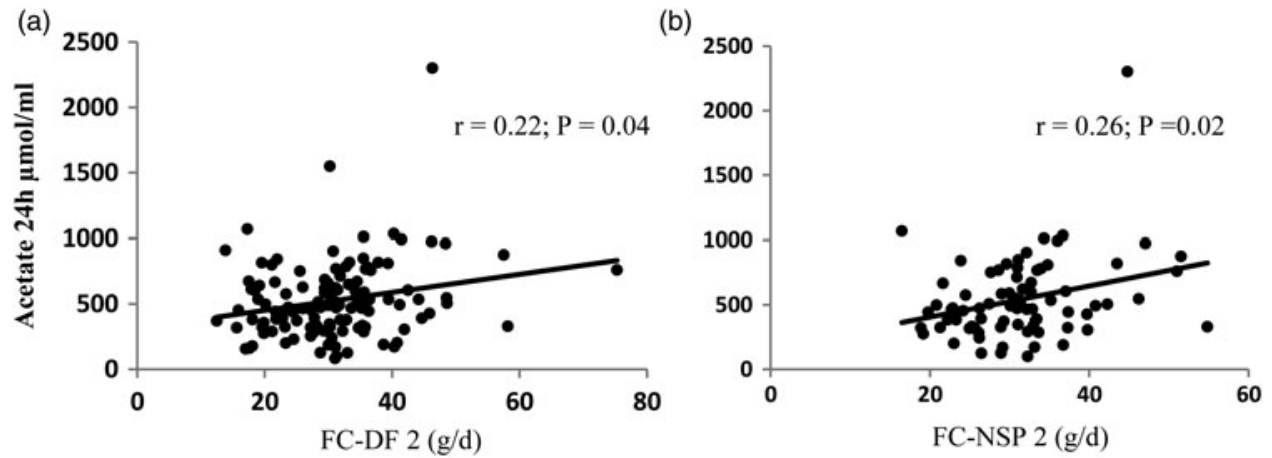

Fig. 1. Correlation of FC-DF2 (A) and FC-NSP2 (B) and $24 \mathrm{~h}$ urinary excretion of acetate

Weak but significant correlations were found for the equations estimating FC. FC-DF2 and FC-NSP2 had better correlations than DF and NSP alone. The inclusion of 50\% insoluble fibre did not greatly improve the correlations. However, urinary SCFA are not well validated for colonic SCFA production estimation, with other factors such as liver metabolism affecting SCFA levels. These equations need to be tested against other measures of colonic fermentation such as in vitro fermentations.

1. Edwards C, Gibson G, Champ M, Jensen B, Mathers J, Nagengast F, et al. (1996) In vitro method for quantification of the fermentation of starch by human faecal bacteria. Journal of the Science of Food and Agriculture 71(2), 209-17. 